\title{
Zero-Field NMR J-Spectroscopy of Organophosphorus Compounds
}

\author{
Seyma Alcicek,* Piotr Put, Vladimir Kontul, and Szymon Pustelny
}

Cite This: J. Phys. Chem. Lett. 2021, 12, 787-792

Read Online

ABSTRACT: Organophosphorus compounds are a wide and diverse class of chemicals playing a crucial role in living organisms. This aspect has been often investigated using nuclear magnetic resonance (NMR), which provides information about molecular structure and function. In this paper, we report the results of theoretical and experimental studies on basic organophosphorus compounds using zero-field NMR, where spin dynamics are investigated in the absence of a magnetic field with the dominant heteronuclear J-coupling. We demonstrate that the zero-field NMR enables distinguishing the chemicals owing to their unique electronic environment even though their spin systems have the same alphabetic designation. Such information can be obtained just

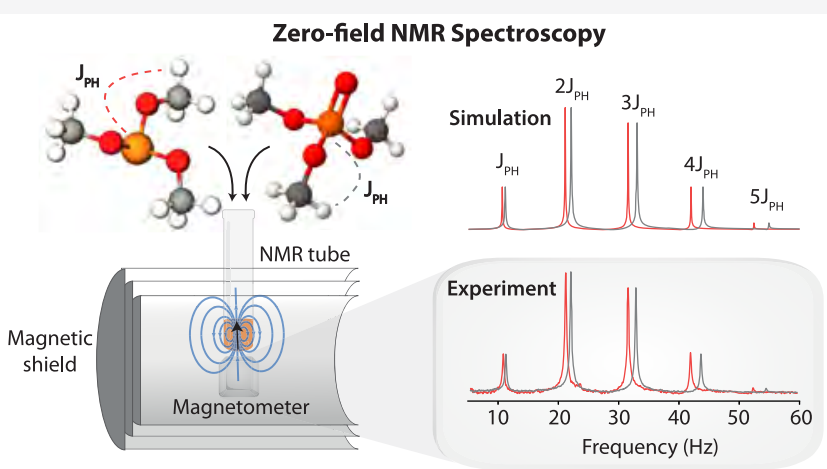
in a single measurement, while amplitudes and widths of observed low-field NMR resonances enable the study of processes affecting spin dynamics. An excellent agreement between simulations and measurements of the spectra, particularly in the largest frequency J-couplings range ever reported in zero-field NMR, is demonstrated.

$\mathrm{I}$ $\mathrm{n}$ recent years, zero-field nuclear magnetic resonance (NMR) has been demonstrated as an alternative NMR modality enabling identification, characterization, and quantification of molecular structures, through observation of unique $J$-coupling information. ${ }^{1-6}$ The basic principle of zero-field NMR spectroscopy is access to indirect spin-spin interactions ( $J$-coupling) due to a vanishing Zeeman interaction in the absence of an applied magnetic field, ${ }^{7,8}$ and averaged out direct spin-spin couplings occurring in isotropic liquids. ${ }^{9}$ Owing to $J$-coupling, yielding useful information about the electronic structure and conformation of molecules, zero-field NMR can be used for chemical analysis and fingerprinting., ${ }^{2,3}$ Zero-field NMR enables the high-resolution measurement of spin-spin couplings with remarkably narrow resonance line widths as a result of the high absolute field homogeneity and long spin coherence times. ${ }^{10,11}$ Furthermore, the greater skin depth at low frequencies enables measurement of samples and monitorization of chemical reactions inside metal containers. ${ }^{12}$ Since in zero-field NMR spin dynamics occurs up to $1000 \mathrm{~Hz}$, an inductive detector is replaced by a sensor sensitive in a near DC frequency range. Atomic magnetometers are often used for this purpose with the main advantages being low-price, small size, and noncryogenic operation, while the ever-growing commercial availability of such sensors leads to technical simplicity and near-zero maintenance. ${ }^{13,14}$ While the zero-field $J$-spectroscopy has been successfully used for thermal studies of isotopically enriched compounds containing ${ }^{13} \mathrm{C},{ }^{15} \mathrm{~N}$ nuclei, here we exploit naturally abundant phosphorus-31 $\left({ }^{31} \mathrm{P}\right)$ nucleus.
Natural phosphorus-containing compounds play vital roles in cell structure, energy metabolism, $\mathrm{pH}$ homeostasis, bone mineralization, etc. ${ }^{15}$ On the other hand, some of the organophosphorus compounds are highly toxic chemicals used as herbicides, insecticides, or fungicides. ${ }^{16,17}$ The naturally abundant isotope of phosphorus $\left({ }^{31} \mathrm{P}\right)$, present in these compounds, is an attractive target for NMR, due to its $100 \%$ natural abundance and spin- $1 / 2 .{ }^{18}$ The in vivo detection of phosphorus-containing metabolites, including inorganic phosphate, adenosine triphosphate (ATP), and phosphocreatine, by a ${ }^{31} \mathrm{P}$ magnetic-resonance spectroscopy, provides information on intracellular $\mathrm{pH}$, cellular energy metabolism, and the phospholipid metabolism in cells. ${ }^{18,19}$ This type of information is valuable for diagnosis and even prognosis of a tumor, $^{20,21}$ despite presenting some challenges such as a long measurement time and complex postprocessing procedure. ${ }^{22}$

In this paper, we report the results of theoretical and experimental studies on basic organophosphorus compounds using zero-field NMR. In order to investigate the relatively large spin systems, compared to previous $J$-spectra studies, we measured trimethyl phosphate and trimethyl phosphite, whose $J$-spectra can be analyzed and identified using a straightforward

Received: November 28, 2020

Accepted: January 5, 2021

Published: January 7, 2021 
interpretation method. We also measured dichlorvos and dimethyl phosphite to examine slightly more complex molecules and evaluate the efficiency of zero-field NMR for a wide range of $J$-coupling values, which contains the resonances with the largest $J$-coupling values ever reported in zero-field NMR spectroscopy. We show the analysis of their spin system using the first-order perturbation theory. Further, we present the comparison of the simulated and experimental $J$-spectra.

Methods of interpretation of zero-field NMR spectra were introduced in a few studies., ${ }^{3,4,10}$ In a simple $\mathrm{XA}_{n}$ nuclear spin system, composed of a heteronucleus $\mathrm{X}$ and a set of $n$ magnetically equivalent nuclei $\mathrm{A}$, the total nuclear spin Hamiltonian $\hat{\mathcal{H}}$ is dominated by the heteronuclear $J$-coupling interaction of the coupling constant $J_{\mathrm{XA}}$ and is given by

$$
\hat{\mathcal{H}}=\sum_{i}^{n} J_{\mathrm{XA}} \hat{\mathbf{s}} \cdot \hat{\mathbf{I}}_{A, i}=J_{\mathrm{XA}} \hat{\mathbf{s}} \cdot \sum_{i}^{n} \hat{\mathbf{I}}_{A, i}=J_{\mathrm{XA}} \hat{\mathbf{s}} \cdot \hat{\mathbf{I}}_{A}
$$

where $\hat{\mathbf{S}}$ and $\hat{\mathbf{I}}_{A, i}$ are the spin vector operators corresponding to the spin $\mathrm{X}$ and the individual spins $\mathrm{A}$, while $\hat{\mathbf{I}}_{A}$ denotes the total spin of all A nuclei, respectively. Due to the dominant scalar interaction, the spins $\mathbf{I}_{A}$ and $\mathbf{S}$ couple into the total spin $\mathbf{F}, \hat{\mathbf{F}}=$ $\hat{\mathbf{I}}_{A}+\hat{\mathbf{S}}$. Energy levels defined by three quantum numbers $S, I_{A}$, and $F$ are given by

$$
E=\frac{J_{\mathrm{XA}}}{2}\left[F(F+1)-I_{A}\left(I_{A}+1\right)-S(S+1)\right]
$$

where $F$ ranges from $\left|I_{A}-S\right|$ to $I_{A}+S$ by unity. For the $\mathrm{XA}_{n}$ spin system, the observable transitions between the states are found by employing the selection rules: $\Delta I_{A}=0$ and $\Delta F=0$, $\pm 1 .{ }^{1}$ Using these rules one can show that for a system of spins $1 / 2\left(S=I_{A, i}=1 / 2\right)$ the spectrum consists of $(n+1) / 2$ lines at frequencies from $J_{\mathrm{XA}}$ to $(n+1) J_{\mathrm{XA}} / 2$ with $J_{\mathrm{XA}}$ intervals. Analogously, if $n$ is even, the spectrum consists of $n / 2$ lines at frequency from $3 J_{\mathrm{XA}} / 2$ to $(n+1) J_{\mathrm{XA}} / 2$ with $J_{\mathrm{XA}}$ intervals. ${ }^{3}$

Trimethyl phosphate and trimethyl phosphite are $\mathrm{XA}_{9}$ spin systems dominated by the ${ }^{31} \mathrm{P}-{ }^{1} \mathrm{H}$ three-bond $J$-coupling (due to low abundance, the effect of the ${ }^{13} \mathrm{C}$ nuclei is negligible) (Figure 1a). Since all ${ }^{1} \mathrm{H}$ nuclei are magnetically equivalent in these compounds, using the rules described above, the frequencies of the allowed transitions and hence the NMR line positions are $J_{\mathrm{PH}}, 2 J_{\mathrm{PH}}, 3 J_{\mathrm{PH}}, 4 J_{\mathrm{PH}}$, and $5 J_{\mathrm{PH}}$ (Figure $1 \mathrm{~b}$ ). Yet, due to their unique electronic environment, the $J_{\mathrm{PH}^{-}}$ coupling values of trimethyl phosphate and trimethyl phosphite are slightly different ( 11 and $10.5 \mathrm{~Hz}$, respectively), and hence the resonances are observed at slightly different frequencies (Figure 1c). The experimental spectrum agrees well with the results of the simulations (Figure 1c,d). Owing to narrow line width, all lines are clearly distinguished in the spectra, allowing for chemical differentiation of these compounds despite constituting the spin systems with the same alphabetical designation, $\mathrm{XA}_{9}$.

More complex spin systems, $\left(\mathrm{XA}_{n}\right) \mathrm{B}_{m}$, contain a heteronuclear $(\mathrm{X})$ and two sets of equivalent nuclei $(n$ number of $\mathrm{A}$ nuclei and $m$ number of $\mathrm{B}$ nuclei) with a stronger $J_{X A}$ coupling compared to the other couplings $\left(J_{X B}, J_{A B}\right)$. The $J$-spectra of such spin systems can be explained using the perturbation theory. The gist of it is that the weaker couplings, by causing energy level shifts, lead to splitting main spectral lines arising from the stronger $J$-coupling interaction. ${ }^{3}$ To examine the $\left(\mathrm{XA}_{n}\right) \mathrm{B}_{m}$ spin systems in more detail, we define additional spin operators which are the spin vector operator of all $\mathrm{B}$ nuclei

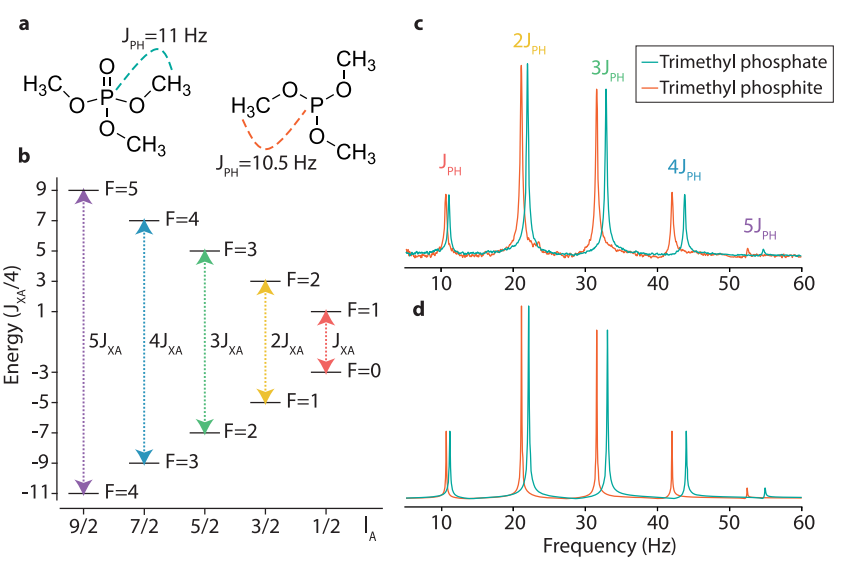

Figure 1. (a) Structural formula and heteronuclear J-coupling interactions in trimethyl phosphate (left) and trimethyl phosphite (right). (b) Energy levels and observable transitions for a $\mathrm{XA}_{9}$ spin system. The manifolds are grouped by the quantum number $I_{A}$, and each manifold is labeled by its quantum number $F$. For clarity, only a single magnetic sublevel is shown in a manifold and just one transition between two states is marked. (c) Experimental and (d) simulated $J$ spectra of trimethyl phosphate and trimethyl phosphite. The experimental spectra are the result of 128 averaged transients.

$\left(\hat{\mathbf{I}}_{B}=\sum_{i}^{n} \hat{\mathbf{I}}_{B, i}\right)$ and total spin operator $\left(\hat{\mathbf{F}}_{T}=\hat{\mathbf{I}}_{B}+\hat{\mathbf{F}}\right)$ with $F_{T}$ being a total-spin quantum number ranging from $\left|I_{B}-F\right|, \mid I_{B}-$ $F \mid+1, \ldots, I_{B}+F-1, I_{B}+F$. As shown in refs 1,3 , and 4 using these spin quantum numbers, the first-order perturbation theory energy shift $\left(E^{1}\right)$ from a zero-order energy level can be derived as

$$
E^{1}=\frac{J_{\mathrm{XB}}^{1}+J_{\mathrm{AB}}^{1}}{2}\left[F_{T}\left(F_{T}+1\right)-F(F+1)-I_{B}\left(I_{B}+1\right)\right]
$$

where $J_{\mathrm{XB}}^{1}$ and $J_{\mathrm{AB}}^{1}$ represent scaled $J$-coupling constants that can be written as

$$
\begin{aligned}
& J_{\mathrm{XB}}^{1}=\frac{J_{\mathrm{XB}}}{2}\left[1+\frac{S(S+1)-I_{A}\left(I_{A}+1\right)}{F(F+1)}\right] \\
& J_{\mathrm{AB}}^{1}=\frac{J_{\mathrm{AB}}}{2}\left[1+\frac{I_{A}\left(I_{A}+1\right)-S(S+1)}{F(F+1)}\right]
\end{aligned}
$$

To find observable transitions between shifted energy levels, we employ the following selection rules: $\Delta I_{A}=\Delta I_{B}=0, \Delta F_{T}=$ $0, \pm 1,3$ which allows us to approximately determine the position of the split lines. To predict the frequency of the lines more precisely, the higher-order terms must be included. This is especially true in cases where closely spaced spectral lines correspond to transitions that are degenerate to first-order. The spectral lines with a distance of up to $0.1 \mathrm{~Hz}$, degenerate in first order perturbation theory, can be distinguished by including second-order corrections or by exact numerical diagonalization as shown in ref 4 . However, the first-order perturbative calculation can provide an intuitive, qualitative interpretation of zero-field spectra. As examples of $\left(\mathrm{XA}_{n}\right) \mathrm{B}_{m}$ spin systems, two organophosphorus compounds, dichlorvos and dimethyl phosphite, were investigated using zero-field NMR. Their molecular structural and relevant $J$-couplings are shown in Figure 2, while below we present the detailed discussion of their $J$-spectra. 


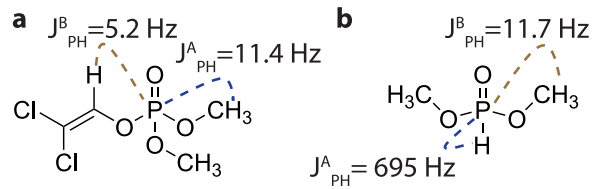

Figure 2. Structural formulas and heteronuclear J-couplings for (a) dichlorvos and (b) dimethyl phosphite investigated as examples of $\left(\mathrm{XA}_{n}\right) \mathrm{B}_{m}$ systems.

Dichlorvos is an example of the $\left(\mathrm{XA}_{6}\right) \mathrm{B}$ spin system, which is composed of one ${ }^{31} \mathrm{P}$ spin, six equivalent ${ }^{1} \mathrm{H}$ spins with three-bond couplings $J_{\mathrm{PH}}^{\mathrm{A}}=11.4 \mathrm{~Hz}$, and another, single ${ }^{1} \mathrm{H}$ spin with a three-bond coupling $J_{\mathrm{PH}}^{\mathrm{B}}=5.2 \mathrm{~Hz}{ }^{17,23}$ A weak $(<0.1$ $\mathrm{Hz}$ ) six-bond homonuclear $J$-coupling between inequivalent ${ }^{1} \mathrm{H}$ nuclei are negligible in this system. In Figure $3 a$, the zero-order energy-level structure is shown. The levels are split and shifted due to weak $J_{X B}$ and the frequencies of the observable transitions are calculated using the first-order perturbation (Figure 3b). The reason for the small, systematic difference $(\approx 0.42 \mathrm{~Hz})$ between estimated frequencies of lines and experimental results is the inadequate separation between the weak and strong $J$-coupling values which reduces the precision of the first-order correction provided by a perturbative calculation. The $\Delta F_{T}= \pm 1$ transitions at lower frequencies $(<3.5 \mathrm{~Hz})$ are not evident in experimental spectra due to spectral overlap ( 6 transitions within a $2-\mathrm{Hz}$ window), short relaxation time, and excessive flicker $(1 / f)$ noise, while the peaks resulting from $\Delta F_{T}=0$ transitions are barely visible in $J$ spectra due to their low relative amplitudes and fast relaxation rate of this compound resulting in a spectral broadening and overlap (Figure 3c). On the other hand, as shown in Figure 3c, a good agreement between the measurement and the numerical simulation is demonstrated. The $\sim 2.5$ times broader line width $(0.85 \mathrm{~Hz})$ in the $J$-spectra of dichlorvos compared to that of trimethyl phosphate is a result of a fast relaxation rate which might be caused by the longer rotational correlation time of this larger compound. ${ }^{24}$ Quadrupolar nuclei, ${ }^{35 / 37} \mathrm{Cl}$, are neglected from spin system analysis, due to their fast relaxations. $^{25}$

Dimethyl phosphite is a $(\mathrm{XA}) \mathrm{B}_{6}$ spin system with a coupling topology similar to that of dichlorvos. The main difference from dichlorvos is the strongest $J$-coupling between ${ }^{31} \mathrm{P}$ and single ${ }^{1} \mathrm{H}$ instead of six equivalent ${ }^{1} \mathrm{H}$ nuclei. The one-bond coupling between ${ }^{1} \mathrm{H}$ and ${ }^{31} \mathrm{P}$ is $J_{\mathrm{PH}}^{\mathrm{A}}=695 \mathrm{~Hz}$, and the three- bond coupling between six ${ }^{1} \mathrm{H}$ and ${ }^{31} \mathrm{P}$ equals $J_{\mathrm{PH}}^{\mathrm{B}}=11.7$ Hz. ${ }^{17,26}$ The splitting of the zero-order energy levels for XA and (XA) $B_{6}$ spin systems is demonstrated in Figure 4a,b. Weak
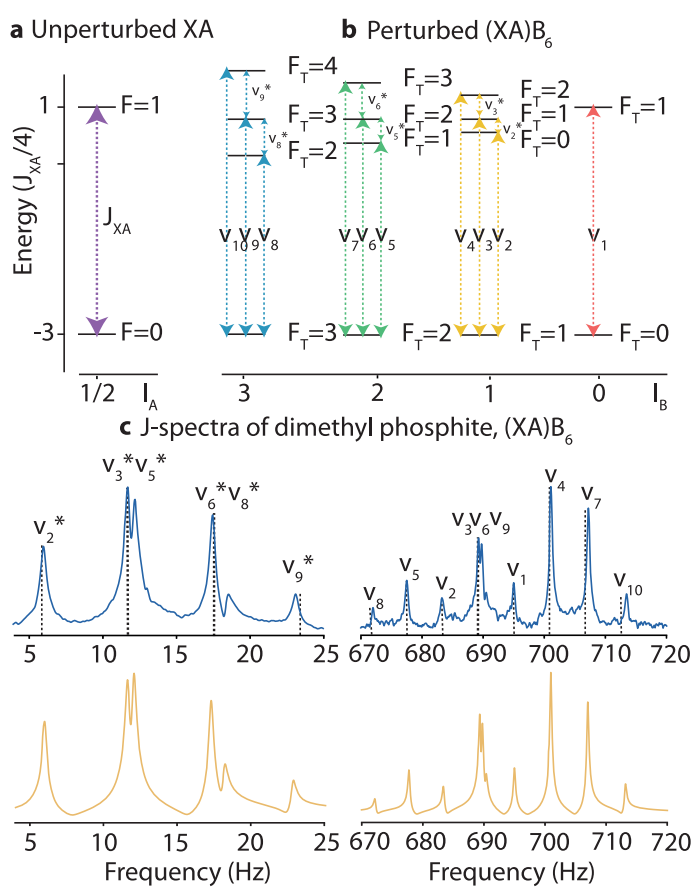

Figure 4. Schematic energy-level structure of the (a) XA and (b) (XA) $B_{6}$ spin system with observable transitions (dashed arrows). The high- and low-frequency transitions are denoted by $\mathrm{v}_{1-10}$ and $\mathrm{v}_{2-9}^{*}$, respectively. The manifolds are grouped by the quantum numbers $I_{A}$ and $I_{B}$, and each manifold is labeled by its quantum number $F$ or $F_{T}$. (c) Experimental spectra (solid blue line), transition frequencies predicted by a first-order perturbation theory (black dashed lines), and simulated zero-field spectra (solid yellow line) for dimethyl phosphite. The experimental spectrum is the result of 128 averaged transients.

four-bond homonuclear $J$-couplings $(<0.5 \mathrm{~Hz})$ between inequivalent ${ }^{1} \mathrm{H}$ nuclei are neglected in this analysis. The $J$ spectrum of dimethyl phosphite can be split into two frequency ranges which arise from the presence of weak and strong couplings in the molecule. The high-frequency $(670-720 \mathrm{~Hz})$ transitions represented as $\mathrm{v}_{1-10}$ and the low-frequency (5-25

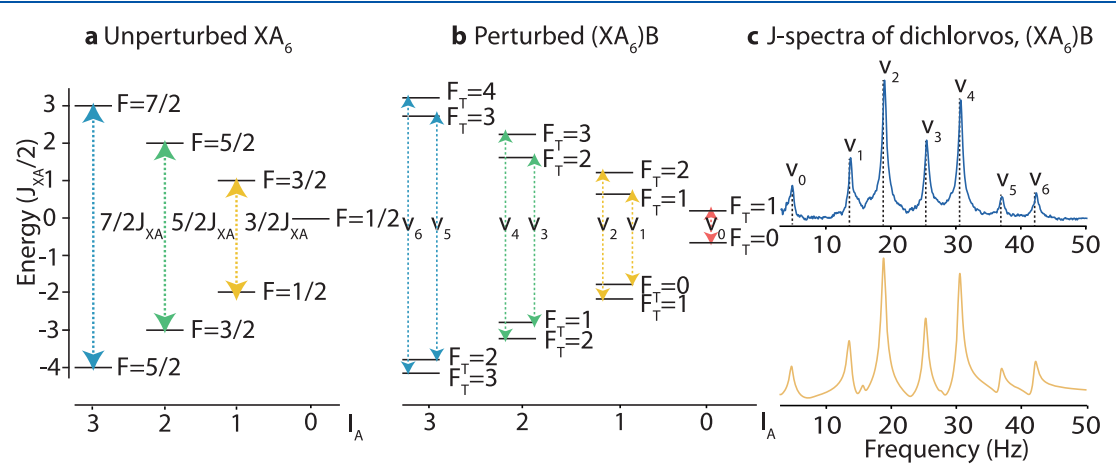

Figure 3. Schematic energy level structure for $(\mathrm{a}) \mathrm{XA}_{6}$ to $(\mathrm{b})\left(\mathrm{XA}_{6}\right) \mathrm{B}$ spin systems and related observable transitions. For a perturbed $\mathrm{XA}_{6}$ spin system, $\mathrm{v}_{0-6}$ denotes the high frequency $(>3.5 \mathrm{~Hz}) \Delta F_{T}= \pm 1$ transitions. The manifolds are grouped by quantum number $I_{A}$, and each manifold is labeled by its quantum number $F$ or $F_{T}$. Only a single sublevel in each manifold and a single transition at each frequency are shown for clarity. (c) Experimental spectra (blue solid line), transition frequencies $\left(\mathrm{v}_{0-6}\right)$ predicted by a first-order perturbation theory (black dashed lines), and the simulated zero-field spectra (solid yellow line) for dichlorvos. The experimental spectrum is the result of 128 averaged transients. 


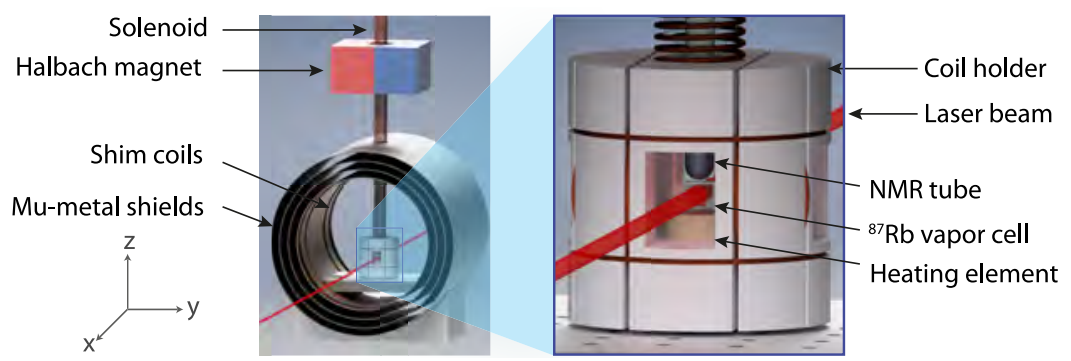

Figure 5. Schematics of the experimental setup. After thermal prepolarization in a 1.8-T Halbach magnet, a sample is transferred to the magnetic shield, to the vicinity of the ${ }^{87} \mathrm{Rb}$ vapor cell. The $Z$-component of the magnetic field, originating from the sample, is measured by monitoring the intensity of light traversing a vapor cell.

$\mathrm{Hz}$ ) transitions represented as $\mathrm{v}_{2-9}^{*}$ were calculated using the $J$ coupling values given above (Figure $4 \mathrm{c}$ ). It is noteworthy that the first-order calculation still produces systematic lines shifts, and more notably does not reproduce additional line splitting seen both in the experiment and exact numerical simulations (Figure 4c). The spectrum of dimethyl phosphite is the zerofield $J$-spectrum with the highest frequency range reported to date. After averaging 128 transients, a signal-to-noise ratios (SNRs) of the strongest line in the $J$-spectrum in low- and high-frequency ranges are $\sim 100$ and $\sim 25$, respectively, stemming from a limited response of the sensor above its bandwidth. The simulated and experimental $J$-spectra yet again present an adequate agreement at both low and high frequencies (Figure 4c).

A major limitation of measurements presented in this study is the low thermal polarization provided by the permanent magnet resulting in low amplitudes of the observed lines. This leads to the use of highly concentrated samples or, in many cases, long measurement times (averages of many transients) to obtain a sufficient SNR. For instance, the detectable concentration limit of the molecules in this study is about $1 \mathrm{M}$ in a single scan. To observe zero-field spectra of diluted phosphorus compounds, e.g., such as those present in biological samples, this limitation needs to be overcome. The hyperpolarization methods such as parahydrogen-induced polarization (PHIP), 27,28 signal amplification by reversible exchange (SABRE) ${ }^{14,29}$ dynamic nuclear polarization (DNP), ${ }^{5}$ etc., which already have been used in zero-field NMR studies, have the potential to facilitate the observation of the important molecules in living cell metabolism. ${ }^{30-32}$ The use of hyperpolarization methods, providing about 4 orders of magnitude signal enhancement, reduces the detectable concentration limit to under the millimolar level. ${ }^{29}$ The hyperpolarization of ${ }^{31} \mathrm{P}$ nuclei in phosphocreatine and inorganic phosphate has been shown in physiological aqueous solution via dissolution DNP. ${ }^{33}$ In addition, ${ }^{31} \mathrm{P}$ hyperpolarization in a phosphine, a phosphine oxide, a phosphine sulfide, and a phosphonate ester has been achieved via SABRE, resulting in a substantial signal enhancement. $^{34,35}$ Another constraint is the finite magnetometer bandwidth, which reduces the sensitivity of the measurements of the phosphorus compounds with stronger one-bond ${ }^{31} \mathrm{P}-{ }^{1} \mathrm{H} J$-couplings, as shown in Figure 4c. This limitation may be lifted by detecting an oscillating field in a given frequency range. Several atomic-magnetometer schemes can detect oscillating magnetic fields in a tunable frequency range. ${ }^{36,37}$

We investigated organophosphorus compounds using a new modality of NMR, zero-field $J$-spectroscopy. We analyzed $J$ spectra of basic organophosphorus molecules using straightfor- ward interpretation methods and demonstrated the ability of chemical identification of compounds forming spin systems of the same alphabetical designation with only small differences in the J-coupling constants. In addition to the first zero-field spectra for ${ }^{31} \mathrm{P}-{ }^{31} \mathrm{H}$ systems, we presented the $J$-spectrum in the broadest frequency range ever reported in zero-field NMR, which enabled us to study the chemicals with weak and strong $J$-coupling interactions simultaneously. Strong agreement between high-spectral-resolution measurements and simulations confirmed that the zero-field NMR can be used for chemical fingerprinting of phosphorus compounds. The analysis and identification of more complex molecules are the subjects of ongoing research. Zero-field NMR techniques, with their promise of portability and low-cost, combined with the ability (shown here) to study naturally abundant phosphorus compounds, can be attractive for the cell metabolism studies. In particular, we plan to use it either to assess energy metabolism via measurement of the important phosphorylated metabolites such as ATP, phosphocreatine, and glucose-phosphate or to evaluate tumor differentiation by analysis of phospholipids. ${ }^{20,22,38,39}$

Trimethyl phosphate (CAS\# 512-56-1), trimethyl phosphite (CAS\# 121-45-9), dimethyl phosphite (CAS\# 868-85-9), and dichlorvos (CAS\# 62-73-7) were sourced from Sigma-Aldrich. The neat liquid samples $(0.15-0.2 \mathrm{~mL})$ were transferred into standard $5 \mathrm{~mm}$ NMR tubes and degassed multiple times using the freeze-pump-thaw method, until the pressure over the frozen liquid reached $<10^{-4}$ mbar. Then the tubes were flamesealed under a vacuum.

A single-beam spin-exchange relaxation free (SERF) atomic magnetometer is used for zero-field NMR measurements. A schematic of our zero-field NMR setup is shown in Figure 5. The three-layer mu-metal shields are used to attenuate the external magnetic field while a ferrite shield (not shown in Figure 5) is used as an innermost layer to reduce magnetic noise, originating from thermal Johnson currents induced in mu-metal. An absolute-zero-magnetic-field region is provided by a set of coils located within the shielding. The field shimming at the position of the NMR sample was performed using a neat ${ }^{13} \mathrm{C}$-formic acid ZULF signal by minimizing splitting of a single $J$-spectrum line. A rubidium-87 $\left({ }^{87} \mathrm{Rb}\right)$ vapor cell $\left(3 \times 3 \times 3 \mathrm{~mm}^{3}\right)$, containing about 300 Torr of $\mathrm{N}_{2}$ as a buffer gas, is placed on a resistively heated ceramic element. The temperature of the cell is held at $160{ }^{\circ} \mathrm{C}$ to preserve sufficient alkali vapor density and operate in the SERF regime. ${ }^{40}$ To optically pump and probe the alkali polarization and hence measure the magnetic field, circularly polarized, resonant $(795 \mathrm{~nm})$ light is used to illuminate the cell and its intensity after passing through the cell is measured by a 
photodiode. In the setup used in this work, the magnetic field is modulated along the $z$-direction at high-frequency $(14 \mathrm{kHz})$ while the photodiode signal is demodulated at this frequency. The phase-sensitive signal from a lock-in is used for magnetometric purposes, with the $z$-axis being a sensitive direction. The magnetometric sensitivity of a setup presented in this work reaches a $60 \mathrm{fT} / \sqrt{\mathrm{Hz}}$ level, while the measurement bandwidth is above $400 \mathrm{~Hz}$ (sensitivity drops significantly above the frequency).

The samples are thermally polarized for $20 \mathrm{~s}$ using a $1.8-\mathrm{T}$ Halbach magnet ${ }^{41}$ placed above the magnetic shield and mechanically shuttled into a zero-field detection region (inside the shield). During the transfer, taking $300 \mathrm{~ms}$, a guiding field of roughly $100 \mu \mathrm{T}$ is applied by a solenoid coil. When the sample reaches the detection area the guiding field is turned off suddenly to generate an oscillating NMR signal. ${ }^{7}$ As a complementary approach, a sharp transverse DC magneticfield pulse along the $y$ axis following turning off the solenoid is used to obtain signals with a higher amplitude (see Supporting Information). Pulses were calibrated using an ultralow field $(\sim 50 \mathrm{nT})$ proton precession signal from a water sample.

A signal-baseline drift present in the magnetometer readout is removed by subtracting a high-order polynomial from the signal, while a digital notch filter has been used to remove 50 $\mathrm{Hz}$ magnetic noise and its overtones. Due to the finite bandwidth of the magnetometer, an initial recuperation period of approximately $100 \mathrm{~ms}$ of the signal has to be removed, while missing data points are replaced by backward prediction using Burg's autoregressive all-pole model method. ${ }^{42}$ To correct for the nonoptimal acquisition time and minimize spectral leakage, the signal is apodized by decaying exponential and then zeropadded to ensure sufficient spectral resolution. The last step of data processing consists of an automatic phase correction using an ACMA algorithm. ${ }^{43}$ The entire data processing is done using Python. Supporting zero-field NMR spectra simulations are performed by a numerical diagonalization of the density matrix, using the high-performance library for spin simulations. ${ }^{10,44}$ For the spin simulations, the $J$-coupling values are taken directly from the literature, providing a match between simulations and experimental data.

\section{ASSOCIATED CONTENT}

\section{s) Supporting Information}

The Supporting Information is available free of charge at https://pubs.acs.org/doi/10.1021/acs.jpclett.0c03532.

Detailed description of generating zero-field NMR signal for ${ }^{31} \mathrm{P}-{ }^{1} \mathrm{H}$ systems and detailed results of the first-order perturbation approach studied (PDF)

\section{AUTHOR INFORMATION}

\section{Corresponding Author}

Seyma Alcicek - Institute of Physics, Faculty of Physics, Astronomy and Applied Computer Science, Jagiellonian University in Kraków, Kraków 30-348, Poland; (1) orcid.org/0000-0002-9447-4906;

Email: seyma.alcicek@uj.edu.pl

\section{Authors}

Piotr Put - Institute of Physics, Faculty of Physics, Astronomy and Applied Computer Science, Jagiellonian University in Kraków, Kraków 30-348, Poland
Vladimir Kontul - Institute of Physics, Faculty of Physics, Astronomy and Applied Computer Science, Jagiellonian University in Kraków, Kraków 30-348, Poland

Szymon Pustelny - Institute of Physics, Faculty of Physics, Astronomy and Applied Computer Science, Jagiellonian University in Kraków, Kraków 30-348, Poland

Complete contact information is available at:

https://pubs.acs.org/10.1021/acs.jpclett.0c03532

\section{Notes}

The authors declare no competing financial interest.

\section{ACKNOWLEDGMENTS}

The authors thank Danila Barskiy, Martyna Elas, Stefan Gloggler, Svenja Knappe, Malcolm H. Levitt, Przemysław Płonka, and Michael Tayler for fruitful discussions. The authors acknowledge the support from the European Union's Horizon 2020 research and innovation program under the Marie Skłodowska-Curie Grant Agreement No. 766402. S.P. acknowledges the support from the grants of the National Science Centre, Poland, within the OPUS program (Project No. 2015/19/B/ST2/02129).

\section{REFERENCES}

(1) Ledbetter, M.; Crawford, C.; Pines, A.; Wemmer, D.; Knappe, S.; Kitching, J.; Budker, D. Optical detection of NMR J-spectra at zero magnetic field. J. Magn. Reson. 2009, 199, 25-29.

(2) Blanchard, J. W.; Ledbetter, M. P.; Theis, T.; Butler, M. C.; Budker, D.; Pines, A. High-Resolution Zero-Field NMR J-Spectroscopy of Aromatic Compounds. J. Am. Chem. Soc. 2013, 135, 36073612.

(3) Theis, T.; Blanchard, J. W.; Butler, M. C.; Ledbetter, M. P.; Budker, D.; Pines, A. Chemical analysis using J-coupling multiplets in zero-field NMR. Chem. Phys. Lett. 2013, 580, 160-165.

(4) Butler, M. C.; Ledbetter, M. P.; Theis, T.; Blanchard, J. W.; Budker, D.; Pines, A. Multiplets at zero magnetic field: The geometry of zero-field NMR. J. Chem. Phys. 2013, 138, 184202.

(5) Barskiy, D.; Tayler, M.; Marco-Rius, I.; Kurhanewicz, J.; Vigneron, D.; Cikrikci, S.; Aydogdu, A.; Reh, M.; Pravdivtsev, A.; Hövener, J.-B.; et al. Zero-field nuclear magnetic resonance of chemically exchanging systems. Nat. Commun. 2019, 10, 3002.

(6) Shimizu, Y.; Blanchard, J.; Pustelny, S.; Saielli, G.; Bagno, A.; Ledbetter, M.; Budker, D.; Pines, A. Zero-field nuclear magnetic resonance spectroscopy of viscous liquids. J. Magn. Reson. 2015, 250, $1-6$.

(7) Blanchard, J. W.; Budker, D. Zero- to Ultralow-field NMR. eMagRes. 2016, 5, 1395-1410.

(8) Ledbetter, M.; Budker, D. Zero-field nuclear magnetic resonance. Phys. Today 2013, 66, 44.

(9) Levitt, M. Spin dynamics: basics of nuclear magnetic resonance, 2nd ed.; John Wiley \& Sons: 2008.

(10) Wilzewski, A.; Afach, S.; Blanchard, J. W.; Budker, D. Method for Measurement of Spin-Spin Couplings with sub-mHz Precision Using Zero- to Ultralow-Field Nuclear Magnetic Resonance. J. Magn. Reson. 2017, 284, 66-72.

(11) Emondts, M.; Ledbetter, M.; Pustelny, S.; Theis, T.; Patton, B.; Blanchard, J.; Butler, M.; Budker, D.; Pines, A. Long-Lived Heteronuclear Spin-Singlet States in Liquids at a Zero Magnetic field. Phys. Rev. Lett. 2014, 112, 077601.

(12) Burueva, D.; Eills, J.; Blanchard, J.; Garcon, A.; Picazo-Frutos, R.; Kovtunov, K.; Koptyug, I.; Budker, D. Chemical Reaction Monitoring using Zero-Field Nuclear Magnetic Resonance Enables Study of Heterogeneous Samples in Metal Containers. Angew. Chem., Int. Ed. 2020, 59, 17026-17032.

(13) Tayler, M. C. D.; Theis, T.; Sjolander, T. F.; Blanchard, J. W.; Kentner, A.; Pustelny, S.; Pines, A.; Budker, D. Invited Review Article: 
Instrumentation for nuclear magnetic resonance in zero and ultralow magnetic field. Rev. Sci. Instrum. 2017, 88, 091101.

(14) Blanchard, J. W.; Wu, T.; Eills, J.; Hu, Y.; Budker, D. Zero- to Ultralow-Field Nuclear Magnetic Resonance J-Spectroscopy with Commercial Atomic Magnetometers. J. Magn. Reson. 2020, 314, 106723.

(15) Corbridge, D. Phosphorus; CRC Press: Boca Raton, 2013.

(16) Tajti, A.; Keglevich, G. The importance of organophosphorus compounds as biologically active agents: Novel Developments; De Gruyter: 2018; pp $53-65$.

(17) Thomas, L. C. The identification of functional groups in organophosphorus compounds; Academic Press: 1974.

(18) Liu, Y.; Gu, Y.; Yu, X. Assessing tissue metabolism by phosphorous-31 magnetic resonance spectroscopy and imaging: a methodology review. Quantitative Imaging in Medicine and Surgery 2017, 7, 707-716.

(19) Cruz, F.; Cerdán, S. Cells Studied by NMR. Encyclopedia of Spectroscopy and Spectrometry; Academic Press: 2010; pp 212-220.

(20) Cohen, J. S. Phospholipid and Energy Metabolism of Cancer Cells Monitored by ${ }^{31} \mathrm{P}$ Magnetic Resonance Spectroscopy: Possible Clinical Significance. Mayo Clin. Proc. 1988, 63, 1199-1207.

(21) Ross, B. D.; Ben-Yoseph, O.; Chenevert, T. L. In In Vivo Magnetic Resonance Imaging and Spectroscopy: Application to Brain Tumors; Bachelard, H., Ed.; Springer: 1997; pp 145-178.

(22) Ha, D.-H.; Choi, S.; Oh, J.; Yoon, S. K.; Kang, M.; Kim, K.-U. Application of 31P MR spectroscopy to the brain tumors. Korean Journal of Radiology 2013, 14, 477-486.

(23) Tebby, J. C. Handbook of Phosphorus-31 Nuclear Magnetic Resonance Data; CRC Press: Boca Raton, 1991.

(24) Harris, R. K.; McVicker, E. M. Spin-lattice relaxation studies of organophosphorus compounds. J. Chem. Soc., Faraday Trans. 2 1976, 72, 2291-2297.

(25) Tayler, M. C.; Gladden, L. F. Scalar relaxation of NMR transitions at ultralow magnetic field. J. Magn. Reson. 2019, 298, 101106.

(26) Gray, G. A. Carbon-13 nuclear magnetic resonance of organophosphorus compounds. I. Diethyl phosphonates. J. Am. Chem. Soc. 1971, 93, 2132-2140.

(27) Theis, T.; Ganssle, P.; Kervern, G.; Knappe, S.; Kitching, J.; Ledbetter, M. P.; Budker, D.; Pines, A. Parahydrogen enhanced zerofield nuclear magnetic resonance. Nat. Phys. 2011, 7, 571-575.

(28) Butler, M. C.; Kervern, G.; Theis, T.; Ledbetter, M. P.; Ganssle, P. J.; Blanchard, J. W.; Budker, D.; Pines, A. Parahydrogen-induced polarization at zero magnetic field. J. Chem. Phys. 2013, 138, 234201.

(29) Theis, T.; Ledbetter, M. P.; Kervern, G.; Blanchard, J. W.; Ganssle, P. J.; Butler, M. C.; Shin, H. D.; Budker, D.; Pines, A. ZeroField NMR Enhanced by Parahydrogen in Reversible Exchange. J. Am. Chem. Soc. 2012, 134, 3987-3990.

(30) Meier, S.; Jensen, P.; Karlsson, M.; Lerche, M. Hyperpolarized NMR Probes for Biological Assays. Sensors 2014, 14, 1576-1597.

(31) Kovtunov, K.; Pokochueva, E.; Salnikov, O.; Cousin, S.; Kurzbach, D.; Vuichoud, B.; Jannin, S.; Chekmenev, E.; Goodson, B.; Barskiy, D. Hyperpolarized NMR Spectroscopy: d-DNP, PHIP, and SABRE Techniques. Chem. - Asian J. 2018, 13, 1857-1871.

(32) Linnik, I. V.; Rayner, P. J.; Stow, R. A.; Duckett, S. B.; Cheetham, G. M. T. Pharmacokinetics of the SABRE agent 4,6-d2nicotinamide and also nicotinamide in rats following oral and intravenous administration. Eur. J. Pharm. Sci. 2019, 135, 32-37.

(33) Nardi-Schreiber, A.; Gamliel, A.; Harris, T.; Sapir, G.; Sosna, J.; Gomori, J.; Katz-Brull, R. Biochemical phosphates observed using hyperpolarized ${ }^{31} \mathrm{P}$ in physiological aqueous solutions. Nat. Commun. 2017, 8, 341.

(34) Zhivonitko, V.; Skovpin, I.; Koptyug, I. Strong ${ }^{31} \mathrm{P}$ nuclear spin hyperpolarization produced via reversible chemical interaction with parahydrogen. Chem. Commun. 2015, 51, 2506-2509.

(35) Burns, M.; Rayner, P.; Duckett, S.; Green, G.; Highton, L.; Mewis, R. Improving the Hyperpolarization of ${ }^{31} \mathrm{P}$ Nuclei by Synthetic Design. J. Phys. Chem. B 2015, 119, 5020-5027.
(36) Savukov, I. M.; Seltzer, S.; Romalis, M.; Sauer, K. Tunable Atomic Magnetometer for Detection of Radio-Frequency Magnetic Fields. Phys. Rev. Lett. 2005, 95, 063004.

(37) Chalupczak, W.; Godun, R.; Pustelny, S.; Gawlik, W. Room temperature femtotesla radio-frequency atomic magnetometer. Appl. Phys. Lett. 2012, 100, 242401.

(38) Bloch, G.; Chase, J. R.; Avison, M. J.; Shulman, R. G. Vivo ${ }^{31} \mathrm{P}$ NMR measurement of glucose-6-phosphate in the rat muscle after exercise. Magn. Reson. Med. 1993, 30, 347-350.

(39) Segebarth, C. M.; Balériaux, D. F.; Arnold, D. L.; Luyten, P. R.; den Hollander, J. A. MR image-guided P-31 MR spectroscopy in the evaluation of brain tumor treatment. Radiology 1987, 165, 215-219.

(40) Savukov, I.; Seltzer, S. Spin-exchange-relaxation-free (SERF) magnetometers. Optical Magnetometry 2011, 85-103.

(41) Tayler, M. C.; Sakellariou, D. Low-cost, pseudo-Halbach dipole magnets for NMR. J. Magn. Reson. 2017, 277, 143-148.

(42) Burg, J. Maximum Entropy Spectral Analysis. Ph.D. Thesis, Stanford University, Stanford, CA, U.S.A., 1975.

(43) Chen, L.; Weng, Z.; Goh, L.; Garland, M. An efficient algorithm for automatic phase correction of NMR spectra based on entropy minimization. J. Magn. Reson. 2002, 158, 164-168.

(44) Afach, S. Spintrum. https://git.afach.de/samerafach/Spintrum. 